\title{
INTEGRABILITY OF (NON-)LINEAR ROUGH DIFFERENTIAL EQUATIONS AND INTEGRALS.
}

\author{
PETER FRIZ, SEBASTIAN RIEDEL
}

\begin{abstract}
Integrability properties of (classical, linear, linear growth) rough differential equations (RDEs) are considered, the Jacobian of the RDE flow driven by Gaussian signals being a motivating example. We revisit and extend some recent ground-breaking work of Cass-LittererLyons in this regard; as by-product, we obtain a user-friendly "transitivity property" of such integrability estimates. We also consider rough integrals; as a novel application, uniform Weibull tail estimates for a class of (random) rough integrals are obtained. A concrete example arises from the stochastic heat-equation, spatially mollified by hyper-viscosity, and we can recover (in fact: sharpen) a technical key result of [Hairer, Comm.PureAppl.Math.64,no.11,(2011),1547-1585].
\end{abstract}

\section{CONTEnTs}

1. Introduction

2. Basis definitions

3. Cass, Litterer and Lyons revisited

4. Transitivity of the tail estimates under locally linear maps 10

4.1. Full RDEs

4.2. Rough integrals

5. Linear RDEs 12

6. Applications in stochastic analysis 14

6.1. Tail estimates for stochastic integrals and solutions of SDEs driven by Gaussian signals 14

6.2. The Jacobian of the solution flow for SDEs driven by Gaussian signals 16

6.3. An example from rough SPDE theory 17

References

\section{INTRODUCTION}

Integrability properties of linear rough differential equations (RDEs), and related topics, driven by Brownian and then a Gaussian rough path (GRP), a random rough path $\mathbf{X}=\mathbf{X}(\omega)$, have been a serious difficulty in a variety of recent applications of rough path theory. To wit, for solutions of linear RDEs one has the typical - and as such sharp - estimate $O\left(\exp \left((\right.\right.$ const $\left.\left.) \times \omega_{\mathbf{x}}(0, T)\right)\right)$ where $\omega_{\mathbf{x}}(0, T)=\|\mathbf{x}\|_{p \text {-var; }[0, T]}^{p}$ denotes some (homogenous) $p$-variation norm (raised to power $p$ ). In a Gaussian rough path setting, $\|\mathbf{X}\|_{p \text {-var; }[0, T]}$ enjoyes Gaussian integrability but as soon as $p>2$ (the "interesting" case, which covers Brownian and rougher situations) one has lost all control over moments of such (random) linear RDE solutions. In a recent work, Cass, Litterer, Lyons [4] have overcome a similar problem, the integrability of the Jacobian of Gaussian RDE flow, as needed in non-Markovian Hörmander theory [14]. 
With these (and some other, cf. below) problems in mind, we revisit the work of Cass, Litterer, Lyons and propose (what we believe to be) a particularly user-friendly formulation. We avoid the concept of "localized p-variation", as introduced in 4, and work throughout with a quantity called $N_{[0, T]}(\mathbf{x})$. As it turns out, in many (deterministic) rough path estimates, as obtained in [12] for instance, one may replace $\omega_{\mathbf{x}}(0, T)$ by $N_{[0, T]}(\mathbf{x})$. Doing so does not require to revisit the (technical) proofs of these rough path estimates, but rather to apply the existing estimates repeatedly on the intervals of a carefully chosen partition of $[0, T]$. The point is that $N_{[0, T]}(\mathbf{X})$ enjoyes much better integrability than $\omega_{\mathbf{X}}(0, T)$. Of course, this does not rule out that for some rough paths $\mathbf{x}, N_{[0, T]}(\mathbf{x}) \approx \omega_{\mathbf{x}}(0, T)$, in agreement with the essentially optimal nature of exisiting rough path estimates in terms of $\omega_{\mathbf{x}}(0, T)$. For instance, both quantities will scale like $\lambda$ when $p=2$ and $\mathbf{x}$ is the pure-area rough path, dilated by $\lambda>>1$. Differently put, the point is that $N_{[0, T]}(\mathbf{X}(\omega))$ will be smaller than $\omega_{\mathbf{X}(\omega)}(0, T)$ for most realizations of $\mathbf{X}(\omega)$.

The consequent focus on $N_{[0, T]}$ rather than "localized $p$-variation" aside, let us briefly enlist our contributions relative to [4].

(i) A technical condition " $p>q[p] "$ is removed; this shows that the Cass, Litterer, Lyons results are valid assuming only "complementary Young regularity of the Cameron-Martin space", i.e. $H \hookrightarrow C^{q-v a r}$ where $1 / p+1 / q>1$ and sample paths have finite $p$-variation, a natural condition, in particular in the context of Malliavin calculus, whose importance was confirmed in a number of papers, 11, [10, 3, 2, see also [12.

(ii) Their technical main result, Weibull tails of $N_{[0, T]}(\mathbf{X})$ with shape parameter $2 / q$, here $\mathbf{X}$ is a Gaussian rough path, remains valid for general rough paths obtained as image of $\mathbf{X}$ under locally linear maps on (rough) path space. (This random rough paths may be far from Gaussian: examples of locally linear maps are given by rough integration and (solving) rough differential equations.)

(iii) The arguments are adapted to deal with (random) linear (and also linear growth) rough differential equations (the solution maps here are not locally linear!) driven by $\mathbf{X}(\omega)$. As above, it suffices that $\mathbf{X}$ is the locally linear image of a Gaussian rough path.

We conclude with two applications. First, we show how to recover $\log$-Weibull tails for $|J|$, the Jacobian of a Gaussian RDE flow. (Afore-mentioned extended validity and some minor sharpening of the norm of $|J|$ aside, this was the main result of [4].) Our point here is that [4 use somewhat involved (known) explicit estimates for the $J$ in terms of the Gaussian driving signal. In contrast, our "user-friendly" formulation allows for a simple step-by-step approach: recall that $J$ solves $d J=$ $J d M$ where $M$ is a non-Gaussian driving rough path, obtained by solving an RDE / performing a rough integration. Since $M$ is the locally linear image of a Gaussian rough path, we can immediately appeal to (iii). As was pointed out recently by [14, such estimates are - in combination with a Norris lemma for rough paths - the key to a non-Markovian Hoermander and then ergodic theory. Secondly, as a novel application, we consider (random) rough integrals of the form $\int G(X) d \mathbf{X}$, with $G \in \operatorname{Lip}^{\gamma-1}, \gamma>p$ and establish Weibull tails with shape parameter 2/q, uniform over classes of Gaussian process whose covariance satisfies a uniform variational estimate. A special case arises when $\mathbf{X}=\mathbf{X}^{\epsilon}$ is taken, independently in each component, as solution to the stochastic heat equation on the $1 \mathrm{D}$ torus, $\dot{u}=u_{x x}+\dot{W}$ with hyper-viscosity term $\epsilon u_{x x x x}$ - as function of the space variable, for fixed time. Complementary Young regularity is seen to hold with $p>2$ and and $q=1$ and we so obtain (and in fact, improve from exponential to Gaussian integrability) the uniform in $\epsilon$ integrability estimate [13, Theorem 5.1, a somewhat central technical result whose proof encompasses almost a third of that paper. 


\section{BASIS DEFINITIONS}

Definition 1. Let $\omega$ be a control. For $\alpha>0$ and $[s, t] \subset[0,1]$ we set

$$
\begin{aligned}
\tau_{0}(\alpha) & =s \\
\tau_{i+1}(\alpha) & =\inf \left\{u: \omega\left(\tau_{i}, u\right) \geq \alpha, \tau_{i}(\alpha)<u \leq t\right\} \wedge t
\end{aligned}
$$

and define

$$
N_{\alpha,[s, t]}(\omega)=\sup \left\{n \in \mathbb{N} \cup\{0\}: \tau_{n}(\alpha)<t\right\} .
$$

When $\omega$ arises from a (homogenous) p-variation norm of a (p-rough) path, such as $\omega_{\mathbf{x}}=\|\mathbf{x}\|_{p \text {-var; } ; \cdot, \cdot]}^{p}$ or $\bar{\omega}_{\mathbf{x}}:=\|\left.|\mathbf{x}|\right|_{p \text {-var; } ; \cdot, \cdot]} ^{p}$, detailed definitions are give later in the text, we shall also write

$$
N_{\alpha,[s, t]}(\mathbf{x}):=N_{\alpha,[s, t]}\left(\omega_{\mathbf{x}}\right) \text { and } \bar{N}_{\alpha,[s, t]}(\mathbf{x}):=N_{\alpha,[s, t]}\left(\bar{\omega}_{\mathbf{x}}\right) \text {. }
$$

In fact, we will be in a situation where $C^{-1} \bar{\omega}_{\mathbf{x}} \leq \omega_{\mathbf{x}} \leq C \bar{\omega}_{\mathbf{x}}$ for some constant $C$ which entails (cf. Lemma 2 below)

$$
\bar{N}_{\alpha C,[\cdot, \cdot]}(\mathbf{x}) \leq N_{\alpha,[\cdot, \cdot]}(\mathbf{x}) \leq \bar{N}_{\alpha / C,[\cdot, \cdot]}(\mathbf{x}) .
$$

Furthermore, the precise value of $\alpha>0$ will not matter (c.f. Lemma 3 below) so that a factor $C$ or $1 / C$ is indeed inconsequential; effectively, this means that one can switch between $N$ and $\bar{N}$ as one pleases.

We now study the scaling of $N_{\alpha}$. Note that $N_{\alpha,[s, t]}(\omega) \searrow 0$ for $\alpha \nearrow \infty$.

Lemma 1. Let $\omega$ be a control and $\lambda>0$. Then $(s, t) \mapsto \lambda \omega(s, t)$ is again a control and for all $s<t$,

$$
N_{\alpha,[s, t]}(\lambda \omega)=N_{\alpha / \lambda,[s, t]}(\omega) .
$$

Proof. Follows directly from the definition.

Lemma 2. Let $\omega_{1}, \omega_{2}$ be two controls, $s<t$ and $\alpha>0$. Assume that $\omega_{1}(u, v) \leq C \omega_{2}(u, v)$ holds whenever $\omega_{2}(u, v) \leq \alpha$ for a constant $C$. Then $N_{C \alpha,[s, t]}\left(\omega_{1}\right) \leq N_{\alpha,[s, t]}\left(\omega_{2}\right)$.

Proof. It suffices to consider the case $C=1$, the general case follows by the scaling of $N$. Set

$$
\begin{aligned}
\tau_{0}^{j}(\alpha) & =s \\
\tau_{i+1}^{j}(\alpha) & =\inf \left\{u: \omega_{j}\left(\tau_{i}^{j}, u\right) \geq \alpha, \tau_{i}^{j}(\alpha)<u \leq t\right\} \wedge t
\end{aligned}
$$

for $j=1,2$. It suffices to show that $\tau_{i}^{2} \leq \tau_{i}^{1}$ holds for every $i \in \mathbb{N}$. By induction over $i$ : For $i=0$ this is clear. If $\tau_{i}^{2} \leq \tau_{i}^{1}$ for some fixed $i$,

$$
\omega_{1}\left(\tau_{i}^{1}, u\right) \leq \omega_{2}\left(\tau_{i}^{1}, u\right) \leq \omega_{2}\left(\tau_{i}^{2}, u\right)
$$

whenever $\omega_{2}\left(\tau_{i}^{2}, u\right) \leq \alpha$. Hence

$$
\inf _{u}\left\{\omega_{2}\left(\tau_{i}^{2}, u\right) \geq \alpha\right\} \leq \inf _{u}\left\{\omega_{1}\left(\tau_{i}^{2}, u\right) \geq \alpha\right\}
$$

and therefore $\tau_{i+1}^{2} \leq \tau_{i+1}^{1}$.

Lemma 3. Let $\omega$ be a control and $0<\alpha \leq \beta$. Then

$$
N_{\alpha,[s, t]}(\omega) \leq \frac{\beta}{\alpha}\left(2 N_{\beta,[s, t]}(\omega)+1\right) .
$$


Proof. Set

$$
\omega_{\alpha}(s, t):=\sup _{\substack{\left(t_{i}\right)=D \subset[s, t] \\ \omega\left(t_{i}, t_{i+1}\right) \leq \alpha}} \sum_{t_{i}} \omega\left(t_{i}, t_{i+1}\right) .
$$

We clearly have $\omega_{\alpha}(s, t) \leq \omega_{\beta}(s, t)$ and

$$
\alpha N_{\alpha,[s, t]}(\omega)=\sum_{i=0}^{N_{\alpha,[s, t]}(\omega)-1} \omega\left(\tau_{i}(\alpha), \tau_{i+1}(\alpha)\right) \leq \omega_{\alpha}(s, t) .
$$

Finally, Proposition 4.6 in 4 ] shows that $\omega_{\beta}(s, t) \leq\left(2 N_{\beta,[s, t]}(\omega)+1\right) \beta$. (Strictly speaking, Proposition 4.6 is formulated for a particular control $\omega$, namely the control induced by the $p$-variation of a rough path. However, the proof only uses general properties of control functions and the conclusion remains valid.)

Let $\mathbf{x}:[0, T] \rightarrow G^{N}\left(\mathbb{R}^{d}\right)$ be a path. In the whole section, $\|\cdot\|_{p-v a r}$ denotes the $p$-variation norm for such paths induced by the Carnot-Caratheodory metric; [12]. Set $\omega_{\mathbf{x}}(s, t)=\|\mathbf{x}\|_{p-v a r ;[s, t]}^{p}$ and $N_{\alpha,[s, t]}(\mathbf{x})=N_{\alpha,[s, t]}\left(\omega_{\mathbf{x}}\right)$ (the fact that $\omega_{\mathbf{x}}$ is indeed a control is well-known; c.f. [12]).

Lemma 4. For any $\alpha>0$,

$$
\|\mathbf{x}\|_{p-v a r ;[s, t]} \leq \alpha^{1 / p}\left(N_{\alpha,[s, t]}(\mathbf{x})+1\right)
$$

Proof. Let $u=u_{0}<u_{1}<\ldots<u_{m}=v$. Note that

$$
\left\|\mathbf{x}_{u, v}\right\|^{p}=\left\|\mathbf{x}_{u, u_{1}} \otimes \mathbf{x}_{u_{1}, u_{2}} \otimes \ldots \otimes \mathbf{x}_{u_{m-1}, v}\right\|^{p} \leq m^{p-1} \sum_{i=0}^{m-1}\left\|\mathbf{x}_{u_{i}, u_{i+1}}\right\|^{p} .
$$

Let $D$ be a dissection of $[s, t]$ and $\left(\tau_{j}\right)_{j=0}^{N_{\alpha,[s, t]}(\mathbf{x})}=\left(\tau_{j}(\alpha)\right)_{j=0}^{N_{\alpha,[s, t]}(\mathbf{x})}$. Set $\bar{D}=D \cup\left(\tau_{j}\right)_{j=0}^{N_{\alpha,[s, t]}(\mathbf{x})}$. Then,

$$
\begin{aligned}
\sum_{t_{i} \in D}\left\|\mathbf{x}_{t_{i}, t_{i+1}}\right\|^{p} & \leq\left(N_{\alpha,[s, t]}(\mathbf{x})+1\right)^{p-1} \sum_{\bar{t}_{i} \in \bar{D}}\left\|\mathbf{x}_{\bar{t}_{i}, \bar{t}_{i+1}}\right\|^{p} \\
& \leq\left(N_{\alpha,[s, t]}(\mathbf{x})+1\right)^{p-1} \sum_{j=0}^{N_{\alpha,[s, t]}(\mathbf{x})}\|\mathbf{x}\|_{p-v a r ;\left[\tau_{j}, \tau_{j+1}\right]}^{p} \\
& \leq\left(N_{\alpha,[s, t]}(\mathbf{x})+1\right)^{p} \alpha .
\end{aligned}
$$

Taking the supremum over all partitions shows the claim.

\section{Cass, Litterer and Lyons Revisited}

The basic object is a continuous $d$-dimensional Gaussian process, say $X$, realized as coordinate process on the (not-too abstract) Wiener space $(E, \mathcal{H}, \mu)$ where $E=C\left([0, T], \mathbb{R}^{d}\right)$ equipped with $\mu$ is a Gaussian measure s.t. $X$ has zero-mean, independent components and that $V_{\rho \text {-var }}\left(R,[0, T]^{2}\right)$, the $\rho$-variation in 2D sense of the covariance $R$ of $X$, is finite for some $\rho \in[1,2)$. From [12, Theorem 15.33] it follows that we can lift the sample paths of $X$ to $p$-rough paths for any $p>2 \rho$ and we denote this process by $\mathbf{X}$, called the enhanced Gaussian process. We also assume that the CameronMartin space $\mathcal{H}$ has complementary Young regularity in the sense that $\mathcal{H}$ embeds continuously in $C^{q \text {-var }}\left([0, T], \mathbb{R}^{d}\right)$ with $\frac{1}{p}+\frac{1}{q}>1$. Note $q \leq p$ for $\mu$ is supported on the paths of finite $p$-variation. 
There are many examples of such a situation [12, let us just note that fractional Brownian motion (fBM) with Hurst parameter $H>1 / 4$ falls in this class of Gaussian rough paths.

In this section, we present, in a self-contained fashion, the results 4 . In fact, we present a slightly modified argument which avoids the technical condition " $p>q[p]$ " made in 4, Theorem 6.2, condition (3)] (this still applies to fBM with $H>1 / 4$ but causes some discontinuities in the resulting estimates when $H$ crosses the barrier 1/3). Our argument also gives a unified treatment for all $p$ thereby clarifying the structure of the proof (in [4, Theorem 6.2] the cases $[p]=2,3$ are treated separately "by hand"). That said, we clearly follow [4] in their ingenious use of Borell's inequality.

In the whole section, if not stated otherwise, for a $p$-rough path $\mathbf{x}$, set

$$
\left.\|\mathbf{x}\|\right|_{p-v a r ;[s, t]}:=\left(\sum_{k=1}^{[p]}\left\|\mathbf{x}^{(k)}\right\|_{p / k-v a r ;[s, t]}^{p / k}\right)^{1 / p} .
$$

Then $\left|\|\cdot \mid\|_{p-v a r}\right.$ is a homogeneous rough path norm. Recall that, as a consequence of Theorem 7.44 in [12], the norms ||$|\cdot| \|_{p-v a r}$ and $\|\cdot\|_{p-v a r}$ are equivalent, hence there is a constant $C$ such that

$$
\frac{1}{C}\||\cdot|\|_{p-v a r} \leq\|\cdot\|_{p-v a r} \leq C\left|\left\|\cdot|\||_{p-v a r} .\right.\right.
$$

The map $(s, t) \mapsto \bar{\omega}_{\mathbf{x}}(s, t)=\||| \mathbf{x} \mid\|_{p-v a r ;[s, t]}^{p}$ is a control and we set $\bar{N}_{\alpha,[s, t]}(\mathbf{x})=N_{\alpha,[s, t]}\left(\bar{\omega}_{\mathbf{x}}\right)$.

Lemma 5. Assume that $\mathcal{H}$ has complementary Young regularity to $X$. Then for any $a>0$, the set

$$
A_{a}=\left\{\mid\|\mathbf{X}\| \|_{p-v a r ;[0, T]}<a\right\}
$$

has positive $\mu$-measure. Moreover, if $M \geq V_{\rho-v a r}\left(R ;[0, T]^{2}\right)$, we have the lower bound

$$
\mu\left\{|||\mathbf{X}| \|_{p-v a r ;[0, T]}<a\right\} \geq 1-\frac{C}{\exp (a)}
$$

where $C$ is a constant only depending on $\rho, p$ and $M$.

Proof. The support theorem for Gaussian rough paths ([12, Theorem 15.60]) shows that

$$
\operatorname{supp}\left[\mathbf{X}_{*} \mu\right]=\overline{S_{[p]}(\mathcal{H})}
$$

holds for $p \in(2 \rho, 4)$. Hence every neighbourhood of the zero-path has positive measure which is the first statement. The general case follows from the a.s. estimate

$$
\left\|\left|S_{\left[p^{\prime}\right]}(\mathbf{X})\right|\right\|_{p^{\prime}-v a r} \leq\left\|\left|S_{\left[p^{\prime}\right]}(\mathbf{X})\right|\right\|_{p-v a r} \leq C_{p, p^{\prime}}\|\mathbf{X} \mid\|_{p-v a r}
$$

which holds for every $p \leq p^{\prime}$, c.f. [12], Theorem 9.5. For the lower bound, recall that from [12], Theorem 15.33 one can deduce that

$$
E\left(\exp || \mathbf{X} \mid \|_{p-v a r ;[0, T]}\right) \leq C
$$

for $p \in(2 \rho, 4)$ where $C$ only depends on $\rho, p$ and $M$. Using (3.2) shows that this actually holds for every $p>2 \rho$. Finally, by Chebychev's inequality,

$$
\mu\left\{\|\mathbf{X} \mid\|_{p-v a r ;[0, T]}<a\right\} \geq 1-\frac{C}{\exp (a)} .
$$


In the next theorem we cite the famous isoperimetric inequality due to C. Borell (for a proof c.f. [16. Theorem 4.3]).

Theorem 1 (Borell). Let $(E, \mathcal{H}, \mu)$ be an abstract Wiener space and $\mathcal{K}$ denote the unit ball in $H$. If $A \subset E$ is a Borell set with positive measure, then for every $r \geq 0$

$$
\mu(A+r \mathcal{K}) \geq \Phi\left(\Phi^{-1}(\mu(A))+r\right)
$$

where $\Phi$ is the cumulative distribution function of a standard normal random variable, i.e. $\Phi=$ $(2 \pi)^{-1 / 2} \int_{-\infty}^{.} \exp \left(-x^{2} / 2\right) d x$.

Corollary 1. Let $f, g: E \rightarrow[0, \infty]$ be measurable maps and $a, \sigma>0$ such that

$$
A_{a}:=\{x: f(x) \leq a\}
$$

has positive measure and let $\hat{a} \leq \Phi^{-1} \mu\left(A_{a}\right)$. Assume furthermore that there exists a null-set $N$ such that for all $x \in N^{c}$ and $h \in \mathcal{H}$ :

$$
f(x-h) \leq a \Rightarrow \sigma\|h\|_{\mathcal{H}} \geq g(x) .
$$

Then $g$ has a Gauss tail; more precisely, for all $r>0$,

$$
\mu(\{x: g(x)>r\}) \leq \exp \left(-\frac{\left(\hat{a}+\frac{r}{\sigma}\right)^{2}}{2}\right) .
$$

Proof. W.l.o.g. $\sigma=1$. Then

$$
\begin{aligned}
\{x: g(x) \leq r\} & =\bigcup_{h \in r \mathcal{K}}\left\{x:\|h\|_{\mathcal{H}} \geq g(x)\right\} \\
& \supset \bigcup_{h \in r \mathcal{K}}\{x: f(x-h) \leq a\} \\
& =\bigcup_{h \in r \mathcal{K}}\{x+h: f(x) \leq a\} \\
& =A_{a}+r \mathcal{K} .
\end{aligned}
$$

By Theorem 1

$$
\mu(\{x: g(x)>r\}) \leq \mu\left(\left\{A_{a}+r \mathcal{K}\right\}^{c}\right) \leq \bar{\Phi}(\hat{a}+r)
$$

where $\bar{\Phi}=1-\Phi$. The claim follows from the standard estimate $\bar{\Phi}(r) \leq \exp \left(-r^{2} / 2\right)$.

Proposition 1. Let $X$ be a continuous d-dimensional Gaussian process, realized as coordinate process on $(E, \mathcal{H}, \mu)$ where $E=C\left([0, T], \mathbb{R}^{d}\right)$ equipped with $\mu$ is a Gaussian measure s.t. $X$ has zero-mean, independent components and that the covariance $R$ of $X$ has finite $\rho$-variationen for some $\rho \in[1,2)$. Let $\mathbf{X}$ be its enhanced Gaussian process with sample paths in a p-rough paths space, $p>2 \rho$. Assume $\mathcal{H}$ has complementary Young regularity, so that Cameron-Martin paths enjoy finite $q$-variation regularity, $q \leq p$ and $\frac{1}{p}+\frac{1}{q}>1$. Then there exists a set $\tilde{E} \subset E$ of full measure with the following property: If

$$
\|\mathbf{X}(\omega-h) \mid\|_{p-v a r ;[0, T]} \leq \alpha^{1 / p}
$$

for all $\omega \in \tilde{E}, h \in \mathcal{H}$ and some $\alpha>0$ then

$$
C|h|_{q-v a r ;[0, T]} \geq \alpha^{1 / p}\left(\bar{N}_{\beta,[0, T]}(\mathbf{X}(\omega))\right)^{1 / q}
$$

where $\beta=2^{p}[p] \alpha$ and $C$ depends only on $p$ and $q$. 
Proof. Set

$$
\tilde{E}=\left\{\omega: T_{h}(\mathbf{X}(\omega))=\mathbf{X}(\omega+h) \text { for all } h \in \mathcal{H}\right\} .
$$

From [12, Lemma 15.58] we know that $\tilde{E}$ has full measure. Define the random partition $\left(\tau_{i}\right)_{i=0}^{\infty}=$ $\left(\tau_{i}(\beta)\right)_{i=0}^{\infty}$ for the control $\bar{\omega}_{\mathbf{X}}$. Let $h \in \mathcal{H}$ and assume that (3.4) holds. We claim that there is a constant $C_{p, q}$ such that

$$
C_{p, q}|h|_{q-v a r ;\left[\tau_{i}, \tau_{i+1}\right]} \geq \alpha^{1 / p} \text { for all } i=0, \ldots, \bar{N}_{\beta,[0, T]}(\mathbf{X})-1 .
$$

The statement then follows from

$$
C_{p, q}^{q}|h|_{q-v a r ;[0, T]}^{q} \geq C_{p, q}^{q} \sum_{i=0}^{\bar{N}_{\beta,[0, T]}(\mathbf{X})-1}|h|_{q-v a r ;\left[\tau_{i}, \tau_{i+1}\right]}^{q} \geq \alpha^{q / p} \bar{N}_{\beta,[0, T]}(\mathbf{X}) .
$$

To show (3.5), we first notice that for every $i=0, \ldots, \bar{N}_{\beta,[0, T]}(\mathbf{X})-1$,

$$
\beta=\left.\|\mathbf{X}(\omega)\|\right|_{p-v a r ;\left[\tau_{i}, \tau_{i+1}\right]} ^{p}=\sum_{k=1}^{[p]}\left\|\mathbf{X}^{(k)}(\omega)\right\|_{p / k-v a r ;\left[\tau_{i}, \tau_{i+1}\right]}^{p / k} .
$$

Fix $i$. Then there is a $k \in\{1, \ldots,[p]\}$ such that $\left\|\mathbf{X}^{(k)}(\omega)\right\|_{p / k-v a r ;\left[\tau_{i}, \tau_{i+1}\right]}^{p / k} \geq \frac{\beta}{p p}$. Let $D=\left(t_{j}\right)_{j=0}^{M}$ be any dissection of $\left[\tau_{i}, \tau_{i+1}\right]$. We define the vector

$$
\mathbf{X}^{(k)}(\omega):=\left(\mathbf{X}^{(k)}(\omega)_{t_{0}, t_{1}}, \ldots, \mathbf{X}^{(k)}(\omega)_{t_{M-1}, t_{M}}\right)
$$

and do the same for $\mathbf{X}^{(k)}(\omega-h)$ and for the mixed iterated integrals

We have then

$$
\int_{\Delta^{k}} d Z^{i_{1}} \otimes \ldots \otimes d Z^{i_{k}} \quad \text { where } Z^{i}=\left\{\begin{array}{ccc}
X & \text { if } & i=0 \\
h & \text { if } & i=1
\end{array} .\right.
$$

$$
\mathbf{X}^{(k)}(\omega-h)=\sum_{\left(i_{1}, \ldots, i_{k}\right) \in\{0,1\}^{k}}(-1)^{i_{1}+\ldots+i_{k}} \int_{\Delta^{k}} d Z^{i_{1}} \otimes \ldots \otimes d Z^{i_{k}}
$$

and by the triangle inequality,

$$
\begin{aligned}
& \left|\int_{\Delta^{k}} d h \otimes \ldots \otimes d h\right|_{l^{p / k}} \\
\geq & \left|\mathbf{X}^{(k)}(\omega)\right|_{l^{p / k}}-\left(\left|\mathbf{X}^{(k)}(\omega-h)\right|_{l^{p / k}}+\sum_{\substack{\left(i_{1}, \ldots, i_{k}\right) \in\{0,1\}^{k} \\
0<i_{1}+\ldots+i_{k}<k}}\left|\int_{\Delta^{k}} d Z^{i_{1}} \otimes \ldots \otimes d Z^{i_{k}}\right|_{l^{p / k}}\right) .
\end{aligned}
$$

Since $q<2$ and $p \geq q$, we can use Young and super-additivity of $|h|_{q-v a r}^{p}$ to see that

$$
\begin{aligned}
\left|\int_{\Delta^{k}} d h \otimes \ldots \otimes d h\right|_{l^{p / k}}^{p / k} & \leq c_{q, k}^{p / k} \sum_{j}|h|_{q-v a r ;\left[t_{j}, t_{j+1}\right]}^{p} \\
& \leq c_{q, k}^{p / k}|h|_{q-v a r ;\left[\tau_{i}, \tau_{i+1}\right]}^{p} .
\end{aligned}
$$

For the mixed integrals one has for any $u<v$

$$
\left|\int_{\Delta_{u, v}^{k}} d Z^{i_{1}} \otimes \ldots \otimes d Z^{i_{k}}\right| \leq c_{k, l, p, q}|h|_{q-v a r ;[u, v]}^{l}|| \mathbf{X}(\omega)||_{p-v a r ;[u, v]}^{k-l}
$$


where $l=i_{1}+\ldots+i_{k}$ (this follows from Theorem 9.26 in [12]). Hence we have, using Hölder's inequality and super-additivity

$$
\begin{aligned}
\left|\int_{\Delta^{k}} d Z^{i_{1}} \otimes \ldots \otimes d Z^{i_{k}}\right|_{l^{p / k}}^{p / k} & \leq c_{k, l, p, q}^{p / k} \sum_{j}|h|_{q-v a r ;\left[t_{j}, t_{j+1}\right]}^{\frac{l p}{k}} \|\left.\mathbf{X}(\omega)||\right|_{p-v a r ;\left[t_{j}, t_{j+1}\right]} ^{\frac{(k-l) p}{k}} \\
& \leq c_{k, l, p, q}^{p / k}\left(\sum_{j}|h|_{q-v a r ;\left[t_{j}, t_{j+1}\right]}^{p}\right)^{l / k}\left(\left.\sum_{j}\|\mathbf{X}(\omega)\|\right|_{p-v a r ;\left[t_{j}, t_{j+1}\right]} ^{p}\right)^{\frac{k-l}{k}} \\
& \leq\left. c_{k, l, p, q}^{p / k}|h|_{q-v a r ;\left[\tau_{i}, \tau_{i+1}\right]}^{\frac{p l}{k}}\|\mathbf{X}(\omega)\|\right|_{p-v a r ;\left[\tau_{i}, \tau_{i+1}\right]} ^{\frac{p(k-l)}{k}}
\end{aligned}
$$

and hence

$$
\begin{aligned}
\left|\int_{\Delta^{k}} d Z^{i_{1}} \otimes \ldots \otimes d Z^{i_{k}}\right|_{l^{p / k}} & \leq c_{k, p, q}|h|_{q-v a r ;\left[\tau_{i}, \tau_{i+1}\right]}^{l}|||\mathbf{X}(\omega)|||_{p-v a r ;\left[\tau_{i}, \tau_{i+1}\right]}^{k-l} \\
& =c_{k, l, p, q}|h|_{q-v a r ;\left[\tau_{i}, \tau_{i+1}\right]}^{l} \beta^{\frac{k-l}{p}} .
\end{aligned}
$$

By assumption,

$$
\left|\mathbf{X}^{(k)}(\omega-h)\right|_{l^{p / k}} \leq\|\mid \mathbf{X}(\omega-h)\|_{p-v a r ;[0, T]}^{k} \leq \alpha^{k / p} .
$$

Plugging this into (3.6) yields

$$
c_{q, k}|h|_{q-v a r ;\left[\tau_{i}, \tau_{i+1}\right]}^{k} \geq\left|\mathbf{X}^{(k)}(\omega)\right|_{l^{p / k}}-\left(\alpha^{k / p}+\sum_{l=1}^{k-1} c_{k, l, p, q}|h|_{q-v a r ;\left[\tau_{i}, \tau_{i+1}\right]}^{l} \beta^{\frac{k-l}{p}}\right) .
$$

Now we can take the supremum over all dissections $D$ and obtain, using $\left\|\mathbf{X}^{(k)}(\omega)\right\|_{p / k-v a r ;\left[\tau_{i}, \tau_{i+1}\right]} \geq$ $\left(\frac{\beta}{[p]}\right)^{k / p}$

$$
\begin{aligned}
c_{q, k}|h|_{q-v a r ;\left[\tau_{i}, \tau_{i+1}\right]}^{k} & \geq\left\|\mathbf{X}^{(k)}(\omega)\right\|_{p / k-v a r ;\left[\tau_{i}, \tau_{i+1}\right]}-\left(\alpha^{k / p}+\sum_{l=1}^{k-1} c_{k, l, p, q}|h|_{q-v a r ;\left[\tau_{i}, \tau_{i+1}\right]}^{l} \beta^{\frac{k-l}{p}}\right) \\
& \geq\left(\frac{\beta}{[p]}\right)^{k / p}-\left(\alpha^{k / p}+\sum_{l=1}^{k-1} c_{k, l, p, q}|h|_{q-v a r ;\left[\tau_{i}, \tau_{i+1}\right]}^{l} \beta^{\frac{k-l}{p}}\right) \\
& =\left(2^{k}-1\right) \alpha^{k / p}-\left(\sum_{l=1}^{k-1}\left(2[p]^{1 / p}\right)^{k-l} c_{k, l, p, q}|h|_{q-v a r ;\left[\tau_{i}, \tau_{i+1}\right]}^{l} \alpha^{\frac{k-l}{p}}\right) .
\end{aligned}
$$

By making constants larger if necessary, we may assume that there is a constant $c_{k, p, q}$ such that

$$
|h|_{q-v a r ;\left[\tau_{i}, \tau_{i+1}\right]}^{k} \geq \frac{\left(2^{k}-1\right)}{c_{k, p, q}} \alpha^{k / p}-\left(\sum_{l=1}^{k-1}|h|_{q-v a r ;\left[\tau_{i}, \tau_{i+1}\right]}^{l} \alpha^{\frac{k-l}{p}}\right) .
$$

This implies that there is a constant $C_{k, p, q}$ depending on $c_{k, p, q}$ such that

$$
C_{k}|h|_{q-v a r ;\left[\tau_{i}, \tau_{i+1}\right]} \geq \alpha^{1 / p} .
$$

Setting $C_{p, q}=\max \left\{C_{1, p, q}, \ldots, C_{[p], p, q}\right\}$ finally shows (3.5).

Now we come to the main result. 
Corollary 2. Let $X$ be a centred Gaussian process in $\mathbb{R}^{d}$ with independent components and covariance $R_{X}$ of finite $\rho$-variation, $\rho<2$. Consider the Gaussian $p$-rough paths $\mathbf{X}$ for $p>2 \rho$ and assume that there is a continuous embedding

$$
\iota: H \hookrightarrow C^{q-v a r}
$$

where $\frac{1}{p}+\frac{1}{q}>1$ and let $K \geq\|\iota\|_{o p}$. Then for every $\alpha>0, \bar{N}_{\alpha,[0, T]}(\mathbf{X})$ has a Weibull tail with shape $2 / q$. More precisely, there is a constant $C=C(p, q)$ such that

$$
\mu\left\{\bar{N}_{\alpha,[0, T]}(\mathbf{X})>r\right\} \leq \exp \left\{-\frac{1}{2}\left(\hat{a}+\frac{\alpha^{1 / p} r^{1 / q}}{C K}\right)^{2}\right\}
$$

for every $r>0$ where $\hat{a}>-\infty$ is chosen such that

$$
\hat{a} \leq \Phi^{-1} \mu\left\{\|\mathbf{X}\|_{p-v a r ;[0, T]}^{p} \leq \frac{\alpha}{2^{p}[p]}\right\} .
$$

Proof. Set

$$
A_{a}=\left\{\omega:\|\mathbf{X}(\omega)\|_{p-v a r ;[0, T]} \leq a^{1 / p}\right\} .
$$

Lemma 5 guarantees that $A_{a}$ has positive measure for any $a>0$. From Proposition 1 we know that there is a set $\tilde{E}$ of full measure such that whenever $\|\mathbf{X}(\omega-h)\|_{p-v a r ;[0, T]} \leq a^{1 / p}$ for $\omega \in \tilde{E}$, $h \in \mathcal{H}$ and $a>0$ we have

$$
a^{1 / p}\left(\bar{N}_{\beta,[0, T]}(\mathbf{X})\right)^{1 / q} \leq c_{p, q}|h|_{q-v a r ;[0, T]} \leq c_{p, q}\|\iota\|_{o p}\|h\|_{\mathcal{H}}
$$

where $\beta=2^{p}[p] a$. Setting $a=\alpha /\left(2^{p}[p]\right)$, Corollary 1 shows that

$$
\mu\left(\left\{\omega: \bar{N}_{\alpha,[0, T]}(\mathbf{X}(\omega))>r\right\}\right) \leq \exp \left(-\left(\frac{\hat{a}}{\sqrt{2}}+\frac{\alpha^{1 / p} r^{1 / q}}{2 \sqrt{2} c_{p, q}[p]^{1 / p}\|\iota\|_{o p}}\right)^{2}\right)
$$

where $\hat{a} \leq \Phi^{-1} \mu\left(A_{a}\right)$.

Remark 1. Corollary Q remains valid if one replaces $\bar{N}_{\alpha,[0, T]}$ by $N_{\alpha,[0, T]}$ in the statement. This follows directly from (3.1) and Lemma 圆 by putting the constant $C$ of (3.1) in the constant $C_{p, q}$ of the respective corollaries.

Remark 2. In [12, Proposition 15.7 is is shown that

$$
|h|_{\rho-v a r} \leq \sqrt{V_{\rho-v a r}\left(R_{X} ;[0, T]^{2}\right)}\|h\|_{\mathcal{H}}
$$

holds for all $h \in \mathcal{H}$. Hence in the regime $\rho \in[1,3 / 2)$ we can always choose $q=\rho$ and the conditions of Corollary Q are fulfilled. For the fractional Brownian motion with Hurst parameter $H$ one can show that $\rho=\frac{1}{2 H}$ and $q>\frac{1}{H+1 / 2}$ are valid choices (cf. [12, chapter 15) and the results of Corollary Q 2 remain valid provided $H>1 / 4$.

Remark 3. If $\mathbf{X}$ is a Gaussian rough paths, we know that $\|\mathbf{X}\|_{p-v a r}$ has a Gaussian tail (or a Weibull tail with shape parameter 2), e.g. obtained by a non-linear Fernique Theorem, cf. [10, whereas Corollary圆 combined with Lemma 4 only gives that $\|\mathbf{X}\|_{p-v a r}$ has a Weibull tail with shape

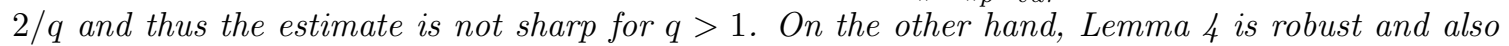
available in situations where Fernique- (or Borell-) type arguments are not directly available, e.g. in a non-Gaussian setting. 


\section{Transitivity of the tail EStimates Under LOCALly LineAR MAPS}

Existing maps for rough integrals and RDE solutions suggest that we consider maps $\Psi$ such that $\|\Psi(\mathbf{x})\|_{p-v a r ; I} \leq$ const. $\|\mathbf{x}\|_{p-v a r ; I}$ uniformly over all intervals $I \subset[0, T]$ where $\|\mathbf{x}\|_{p-v a r ; I} \leq R$ $R>0$. More formally,

Definition 2. We call $\Psi: C^{p-v a r}\left([0, T] ; G^{N}\left(\mathbb{R}^{d}\right)\right) \rightarrow C^{p-v a r}\left([0, T] ; G^{M}\left(\mathbb{R}^{e}\right)\right)$ a locally linear map if there is a $R \in(0, \infty]$ such that

$$
\|\Psi\|_{R}:=\inf _{C>0}\left\{\|\Psi(\mathbf{x})\|_{p-v a r ;[u, v]} \leq C\|\mathbf{x}\|_{p-v a r ;[u, v]} \text { for all }(u, v) \in \Delta, \mathbf{x} \text { s.t. }\|\mathbf{x}\|_{p-v a r ;[u, v]} \leq R\right\}
$$

is finite.

\section{Remark 4. (1)}

(2) For $\lambda \in \mathbb{R}$, we denote by $\delta_{\lambda}$ the dilation map. Set $\left(\delta_{\lambda} \Psi\right): \mathbf{x} \mapsto \delta_{\lambda} \Psi(\mathbf{x})$. Then $\|\cdot\|_{R}$ is homogeneous w.r.t. dilation, e.g. $\left\|\delta_{\lambda} \Psi\right\|_{R}=|\lambda|\|\Psi\|_{R}$.

(3) If $\Psi$ commutes with the dilation map $\delta$ modulo p-variation, e.g. $\left\|\Psi\left(\delta_{\lambda} \mathbf{x}\right)\right\|_{p \text {-var;I }}=\left\|\delta_{\lambda} \Psi(\mathbf{x})\right\|_{p-v a r ; I}$ for any $\mathbf{x}, \lambda \in \mathbb{R}$ and intervall $I \subset[0, T]$, we have $\|\Psi\|_{R}=\|\Psi\|_{\infty}$ for any $R>0$. An example of such a map is the Lyons lift map

$$
S_{N}: C^{p-\operatorname{var}}\left([0, T] ; G^{[p]}\left(\mathbb{R}^{d}\right)\right) \rightarrow C^{p-\operatorname{var}}\left([0, T] ; G^{N}\left(\mathbb{R}^{d}\right)\right)
$$

for which we have $\left\|S_{N}\right\|_{\infty} \leq C(N, p)<\infty$, c.f. [12], Theorem 9.5.

(4) If $\phi:[0, T] \rightarrow \phi[0, T] \subset[0, T]$ is a bijective, continuous and increasing function and $\mathbf{x}$ a rough path, we set $\mathbf{x}_{t}^{\phi}=\mathbf{x}_{\phi(t)}$ and call $\mathbf{x}^{\phi}$ a reparametrization of $\mathbf{x}$. If $\Psi$ commutes with reparametrization modulo p-variation, e.g. $\left\|\Psi\left(\mathbf{x}^{\phi}\right)\right\|_{p-v a r ; I}=\left\|\Psi(\mathbf{x})^{\phi}\right\|_{p-\text { var } ; I}$ for any $\mathbf{x}$, $\phi$ and intervall $I \subset[0, T]$, we have

$$
\|\Psi\|_{R}:=\inf _{C>0}\left\{\|\Psi(\mathbf{x})\|_{p-v a r ;[0, T]} \leq C\|\mathbf{x}\|_{p-v a r ;[0, T]} \text { for all } \mathbf{x} \text { s.t. }\|\mathbf{x}\|_{p-v a r ;[0, T]} \leq R\right\} \text {. }
$$

This follows by a standard reparametrization argument. Examples of such maps are rough integration over 1-forms, e.g. $\mathbf{x} \mapsto \int_{0}^{\cdot} \varphi(x) d \mathbf{x}$, and the Itō-Lyons map, e.g. $\Psi(\mathbf{x})_{s, t}=\mathbf{y}_{s, t}$ where $\mathbf{y}$ solves $d \mathbf{y}=V(\mathbf{x})$ d $\mathbf{x}$ with initial condition $\mathbf{y}_{0} \in G^{[p]}\left(\mathbb{R}^{e}\right)$. In this case,

$$
\|\Psi\|_{\infty}=\sup _{\mathbf{x}} \frac{\|\Psi(\mathbf{x})\|_{p-v a r ;[0, T]}}{\|\mathbf{x}\|_{p-v a r ;[0, T]}}
$$

(where $0 / 0:=0$ ) and we find the usual operator norm. (Note that, however, we can not speak of linear maps in this context since rough paths spaces are typically non-linear.)

(5) Clearly, if $\|\Psi\|_{\infty}<\infty,\|\Psi(\mathbf{X})\|_{p-v a r ;[s, t]}$ inherits the integrability properties of $\|\mathbf{X}\|_{p-v a r ;[s, t]}$. However, for the most interesting maps, e.g. the Ito-Lyons map, we will not have $\|\Psi\|_{\infty}<$ $\infty$, but $\|\Psi\|_{R}<\infty$ for any finite $R>0$. In a way, the purpose of this section is to show that one still has transitivity of integrability if one considers $N_{\alpha,[s, t]}(\mathbf{X})$ instead of $\|\mathbf{X}\|_{p-v a r ;[s, t]}$.

Lemma 6. Let $\Psi$ and $\Phi$ be locally linear maps with $\|\Psi\|_{R}<\infty$ and $\|\Phi\|_{R\|\Psi\|_{R}}<\infty$. Then $\Psi \circ \Phi$ is again locally linear and

$$
\|\Phi \circ \Psi\|_{R} \leq\|\Phi\|_{R\|\Psi\|_{R}}\|\Psi\|_{R} .
$$


Proof. Let $\|\mathbf{x}\|_{p-v a r ;[u, v]} \leq R$. Then $\|\Psi(\mathbf{x})\|_{p-v a r ;[u, v]} \leq\|\Psi\|_{R}\|\mathbf{x}\|_{p-v a r ;[u, v]} \leq R\|\Psi\|_{R}$ which implies

$$
\|\Phi \circ \Psi(\mathbf{x})\|_{p-v a r ;[u, v]} \leq\|\Phi\|_{R\|\Psi\|_{R}}\|\Psi(\mathbf{x})\|_{p-v a r ;[u, v]} \leq\|\Phi\|_{R\|\Psi\|_{R}}\|\Psi\|_{R}\|\mathbf{x}\|_{p-v a r ;[u, v]} .
$$

The interesting property of locally linear maps is formulated in the next proposition.

Proposition 2. Let $\Psi: C^{p-v a r}\left([0, T] ; G^{N}\left(\mathbb{R}^{d}\right)\right) \rightarrow C^{p-v a r}\left([0, T] ; G^{M}\left(\mathbb{R}^{e}\right)\right)$ be locally linear and $\|\Psi\|_{R}<\infty$ for some $R \in(0, \infty]$. Then

$$
N_{\alpha\|\Psi\|_{R}^{p},[s, t]}(\Psi(\mathbf{x})) \leq N_{\alpha,[s, t]}(\mathbf{x})
$$

for any $s<t$ and $\alpha \in\left(0, R^{p}\right]$.

Proof. Follows directly from Lemma 2

4.1. Full RDEs. Consider the full RDE

$$
d \mathbf{y}=V(\mathbf{y}) d \mathbf{x} ; \quad \mathbf{y}_{0} \in G^{[p]}\left(\mathbb{R}^{e}\right)
$$

where $\mathbf{x}$ is a weak geometric $p$-rough path with values in $G^{[p]}\left(\mathbb{R}^{d}\right), V=\left(V_{i}\right)_{i=1, \ldots . d}$ is a collection of $\operatorname{Lip}^{\gamma}$-vector fields in $\mathbb{R}^{e}$ where $\gamma>p$ and $\mathbf{y}_{0}$ is the initial value. Theorem 10.36 and 10.38 in [12] state that (4.1) possesses a unique solution $\mathbf{y}$ which is a weak geometric $p$-rough path with values in $G^{[p]}\left(\mathbb{R}^{e}\right)$.

Corollary 3. The Itō-Lyons map $\Psi: \mathbf{x} \mapsto \mathbf{y}$ is locally linear with

$$
\|\Psi\|_{R} \leq K\left(\|V\|_{L i p^{\gamma-1}} \vee\|V\|_{L i p^{\gamma-1}}^{p} R^{p-1}\right)
$$

for any $R \in(0, \infty)$ where $K$ only depends on $p$ and $\gamma$. Moreover, if $\|V\|_{L i p^{\gamma-1}} \leq \nu$, then for any $\alpha>0$ there is a constant $C=C(p, \gamma, \nu, \alpha)$ such that

$$
N_{\alpha,[s, t]}(\mathbf{y}) \leq C\left(N_{\alpha,[s, t]}(\mathbf{x})+1\right)
$$

for any $s<t$.

Proof. (4.2) follows from the estimate (10.26) of Theorem 10.36 in [12]. From Proposition 2 we obtain

$$
N_{\beta,[s, t]}(\Psi(\mathbf{x})) \leq N_{\alpha,[s, t]}(\mathbf{x})
$$

where $\beta=\alpha\|\Psi\|_{\alpha^{1 / p}}^{p}$. This already shows the claim if $\|\Psi\|_{\alpha^{1 / p}}^{p} \leq 1$. In the case $\|\Psi\|_{\alpha^{1 / p}}^{p}>1$, we conclude with Lemma 3 ,

4.2. Rough integrals. If $\mathbf{x}$ is a $p$-rough path and $\varphi=\left(\varphi_{i}\right)_{i=1, \ldots, d}$ a collection of $\operatorname{Lip}^{\gamma-1}\left(\mathbb{R}^{d}, \mathbb{R}^{e}\right)$ maps, one can define the rough integral

$$
\int \varphi(x) d \mathbf{x}
$$

as an element in $C^{p-v a r}\left([0, T] ; G^{[p]}\left(\mathbb{R}^{e}\right)\right)$ (c.f. [12], chapter 10.6). 
Corollary 4. The map $\Psi: \mathbf{x} \mapsto \mathbf{z}$, $\mathbf{z}$ given by the rough integral (4.3), is locally linear with

$$
\|\Psi\|_{R} \leq K\|\varphi\|_{L i p^{\gamma-1}}\left(1 \vee R^{p-1}\right)
$$

for any $R \in(0, \infty)$ where $K$ only depends on $p$ and $\gamma$. Moreover, if $\|\varphi\|_{L i p^{\gamma-1}} \leq \nu$, then for any $\alpha>0$ there is a constant $C=C(p, \gamma, \nu, \alpha)$ such that

$$
N_{\alpha,[s, t]}(\mathbf{z}) \leq C\left(N_{\alpha,[s, t]}(\mathbf{x})+1\right)
$$

for any $s<t$.

Proof. (4.4) follows from [12, Theorem 10.47. One proceeds as in the proof of Corollary 3 ,

\section{LINEAR RDES}

For a $p$-rough path $\mathbf{x}$, consider the full linear RDE

$$
d \mathbf{y}=V(\mathbf{y}) d \mathbf{x} ; \quad \mathbf{y}_{0} \in G^{[p]}\left(\mathbb{R}^{e}\right)
$$

where $V=\left(V_{i}\right)_{i=1, \ldots, d}$ is a collection of linear vector fields of the form $V_{i}(z)=A_{i} z+b_{i}, A_{i}$ are $e \times e$ matrices and $b_{i} \in \mathbb{R}^{e}$. It is well-known (e.g. [12, section 10.7) that in this case (5.1) has a unique solution $\mathbf{y}$. Unfortunately, the map $\Psi: \mathbf{x} \mapsto \mathbf{y}$ is not locally linear in the sense of Definition 2 and our tools of the former section do not apply. However, we can do a more direct analysis and obtain a different transitivity of the tail estimates.

Let $\nu$ be a bound on $\max _{i}\left(\left|A_{i}\right|+\left|b_{i}\right|\right)$ and set $y=\pi_{1}(\mathbf{y})$. In [12, Theorem 10.53 one sees that there is a constant $C$ depending only on $p$ such that

$$
\left\|\mathbf{y}_{s, t}\right\| \leq C\left(1+\left|y_{s}\right|\right) \nu\|\mathbf{x}\|_{p-v a r ;[s, t]} \exp \left(C \nu^{p}\|\mathbf{x}\|_{p-v a r ;[s, t]}^{p}\right)
$$

holds for all $s<t \in[0, T]$. (Strictly speaking, we only find the estimate for $(s, t)=(0,1)$, the general case follows by reparametrization.) We start with an estimate for the supremum norm of $y$.

Lemma 7. For any $\alpha>0$ there is a constant $C=C(p, \nu, \alpha)$ such that

$$
|y|_{\infty ;[s, t]} \leq C\left(1+\left|y_{s}\right|\right) \exp \left(C N_{\alpha ;[s, t]}(\mathbf{x})\right)
$$

holds for any $s<t$.

Proof. From (5.2) we have

$$
\left|y_{u, v}\right| \leq C\left(1+\left|y_{u}\right|\right) \nu\|\mathbf{x}\|_{p-v a r ;[u, v]} \exp \left(C \nu^{p}\|\mathbf{x}\|_{p-v a r ;[u, v]}^{p}\right)
$$

for any $u<v \in[s, t]$. From $\left|y_{u, v}\right|=\left|y_{s, v}-y_{s, u}\right| \geq\left|y_{s, v}\right|-\left|y_{s, u}\right|$ we obtain

$$
\begin{aligned}
\left|y_{s, v}\right| & \leq C\left(1+\left|y_{u}\right|\right) \nu\|\mathbf{x}\|_{p-v a r ;[u, v]} \exp \left(C \nu^{p}\|\mathbf{x}\|_{p-v a r ;[u, v]}^{p}\right)+\left|y_{s, u}\right| \\
& \leq C\left(1+\left|y_{s}\right|+\left|y_{s, u}\right|\right) \exp \left\{C \nu^{p}\|\mathbf{x}\|_{p-v a r ;[u, v]}^{p}\right\}
\end{aligned}
$$


by making $C$ larger. Now let $s=\tau_{0}<\ldots<\tau_{N}<\tau_{M+1}=u \leq t$ with $M \geq 0$. By induction, one sees that

$$
\begin{aligned}
\left|y_{s, u}\right| & \leq C^{M+1}\left((M+1)\left(1+\left|y_{s}\right|\right)\right) \exp \left\{C \sum_{i=0}^{M} \nu^{p}\|\mathbf{x}\|_{p-v a r ;\left[\tau_{i}, \tau_{i+1}\right]}^{p}\right\} \\
& \leq C^{M+1}\left(1+\left|y_{s}\right|\right) \exp \left\{C \sum_{i=0}^{M} \nu^{p}\|\mathbf{x}\|_{p-v a r ;\left[\tau_{i}, \tau_{i+1}\right]}^{p}\right\} .
\end{aligned}
$$

This shows that for every $u \in[s, t]$,

$$
\begin{aligned}
\left|y_{s, u}\right| & \leq C^{\left(N_{\alpha ;[s, t]}(\mathbf{x})+1\right)}\left(1+\left|y_{s}\right|\right) \exp \left(C \nu^{p} \alpha\left(N_{\alpha ;[s, t]}(\mathbf{x})+1\right)\right) \\
& =\left(1+\left|y_{s}\right|\right) \exp \left\{\left(\log (C)+C \nu^{p} \alpha\right)\left(N_{\alpha ;[s, t]}(\mathbf{x})+1\right)\right\}
\end{aligned}
$$

and hence

$$
\sup _{u \in[s, t]}\left|y_{s, u}\right| \leq C\left(1+\left|y_{s}\right|\right) \exp \left(C N_{\alpha ;[s, t]}(\mathbf{x})\right)
$$

for a constant $C=C(p, \nu, \alpha)$ and therefore also

$$
|y|_{\infty ;[s, t]} \leq C\left(1+\left|y_{s}\right|\right) \exp \left(C N_{\alpha ;[s, t]}(\mathbf{x})\right) .
$$

Corollary 5. Let $\alpha>0$. Then there is a constant $C=C(p, \nu, \alpha)$ such that

$$
N_{\alpha,[s, t]}(\mathbf{y}) \leq C\left(1+\left|y_{s}\right|\right)^{p} \exp \left(C N_{\alpha ;[s, t]}(\mathbf{x})\right)
$$

for any $s<t$.

Proof. Using (5.2) we can deduce that

$$
\left\|\mathbf{y}_{u, v}\right\| \leq C\left(1+|y|_{\infty ;[s, t]}\right) \nu\|\mathbf{x}\|_{p-v a r ;[u, v]} \exp \left(C \nu^{p}\|\mathbf{x}\|_{p-v a r ;[u, v]}^{p}\right)
$$

holds for any $u<v \in[s, t]$ and hence also

$$
\|\mathbf{y}\|_{p-v a r ;[u, v]} \leq C\left(1+|y|_{\infty ;[s, t]}\right) \nu\|\mathbf{x}\|_{p-v a r ;[u, v]} \exp \left(C \nu^{p}\|\mathbf{x}\|_{p-v a r ;[u, v]}^{p}\right)
$$

for any $u<v \in[s, t]$. Now take $u<v \in[s, t]$ such that $\|\mathbf{x}\|_{p-v a r ;[u, v]}^{p} \leq \alpha$. We then have

$$
\|\mathbf{y}\|_{p-v a r ;[u, v]}^{p} \leq \tilde{C}\|\mathbf{x}\|_{p-v a r ;[u, v]}^{p}
$$

where

From Lemma 2

$$
\tilde{C}=C^{p}\left(1+|y|_{\infty ;[s, t]}\right)^{p} \nu^{p} \exp \left(p C \nu^{p} \alpha\right)
$$

$$
N_{\tilde{C} \alpha,[s, t]}(\mathbf{y}) \leq N_{\alpha,[s, t]}(\mathbf{x}) .
$$

If $\tilde{C} \leq 1$, this already shows the claim. For $\tilde{C}>1$, we use Lemma 3 and Lemma 7 to see that

$$
\begin{aligned}
N_{\alpha,[s, t]}(\mathbf{y}) & \leq\left(2 N_{\tilde{C} \alpha,[s, t]}(\mathbf{y})+1\right) \tilde{C} \\
& \leq C\left(N_{\alpha,[s, t]}(\mathbf{x})+1\right)\left(1+|y|_{\infty ;[s, t]}\right)^{p} \\
& \leq C\left(1+\left|y_{s}\right|\right)^{p} \exp \left(C N_{\alpha,[s, t]}(\mathbf{x})\right) .
\end{aligned}
$$


Remark 5 (Unbounded vector fields). Let $\mathbf{x}$ be a p-rough path. Consider a collection $V=\left(V_{i}\right)_{1 \leq i \leq d}$ of locally Lip ${ }^{\gamma-1}$-vector fields on $\mathbb{R}^{e}, \gamma \in(p,[p]+1)$, such that $V_{i}$ are Lipschitz continuous and the vector fields $V^{[p]}=\left(V_{i_{1}}, \ldots, V_{i_{[p]}}\right)_{i_{1}, \ldots, i_{[p]} \in\{1, \ldots, d\}}$ are $(\gamma-[p])$-Hölder continuous. Then the $R D E$

$$
d \mathbf{y}=V(\mathbf{y}) d \mathbf{x} ; \quad \mathbf{y}_{0} \in G^{[p]}\left(\mathbb{R}^{e}\right)
$$

has a unique solution (c.f. [12], Exercise 10.56 and the solution thereafter and [18]). Moreover, in [12] it is shown that

$$
\left\|\mathbf{y}_{0,1}\right\| \leq C\left(1+\left|y_{0}\right|\right) \nu\|\mathbf{x}\|_{p-v a r ;[0,1]} \exp \left(C \nu^{p}\|\mathbf{x}\|_{p-v a r ;[0,1]}^{p}\right)
$$

where $C=C(p, \gamma)$ and $\nu$ is a bound on $\left|V^{[p]}\right|_{(\gamma-[p])-H o ̈ l}^{1 /[p]} \sup _{y, z} \frac{|V(y)-V(z)|}{|y-z|}$. This shows that Lemma 7 and Corollary 5 apply for $\mathbf{y}$, hence for any $\alpha>0$ there is a constant $C=C(p, \gamma, \nu, \alpha)$ such that

$$
N_{\alpha,[s, t]}(\mathbf{y}) \leq C\left(1+\left|y_{s}\right|\right)^{p} \exp \left(C N_{\alpha ;[s, t]}(\mathbf{x})\right)
$$

for all $s<t$ in this case.

\section{Applications in stochastic analysis}

6.1. Tail estimates for stochastic integrals and solutions of SDEs driven by Gaussian signals. We now apply our results to solutions of SDEs and stochastic integrals driven by Gaussian signals, i.e. a Gaussian rough path $\mathbf{X}$. Remark that all results here may be immediately formulated for SDEs and stochastic integrals driven by random rough paths as along as suitable quantitative Weibull-tail estimate for $N_{\alpha,[0, T]}(\mathbf{X})$ are assumed.

We first consider the non-linear case:

Proposition 3. Let $X$ be a centred Gaussian process in $\mathbb{R}^{d}$ with independent components and covariance $R_{X}$ of finite $\rho$-variation, $\rho<2$. Consider the Gaussian $p$-rough paths $\mathbf{X}$ for $p>2 \rho$ and assume that there is a continuous embedding

$$
\iota: H \hookrightarrow C^{q-v a r}
$$

where $\frac{1}{p}+\frac{1}{q}>1$. Let $Y:[0, T] \rightarrow \mathbb{R}^{e}$ be the pathwise solution of the stochastic $R D E$

$$
d Y=V(Y) d \mathbf{X} ; \quad Y_{0} \in \mathbb{R}^{e}
$$

where $V=\left(V_{i}\right)_{i=1, \ldots . d}$ is a collection of Lip ${ }^{\gamma}$-vector fields in $\mathbb{R}^{e}$ with $\gamma>p$. Moreover, let $Z:[0, T] \rightarrow \mathbb{R}^{e}$ be the stochastic integral given by

$$
Z_{t}=\pi_{1}\left(\int_{0}^{t} \varphi(X) d \mathbf{X}\right)
$$

where $\varphi=\left(\varphi_{i}\right)_{i=1, \ldots, d}$ is a collection of Lip ${ }^{\gamma-1}\left(\mathbb{R}^{d}, \mathbb{R}^{e}\right)$-maps, $\gamma>p$. Then both $\|Y\|_{p-v a r ;[0, T]}$ and $\|Z\|_{p-v a r ;[0, T]}$ have Weibull tails with shape parameter $2 / q$. More precisely, if $K \geq\|\iota\|_{o p}$, $M \geq V_{\rho-v a r}\left(R ;[0, T]^{2}\right)$ and $\nu \geq\|V\|_{L^{p^{\gamma-1}}}$ there is a constant $\eta=\eta(p, q, \rho, \gamma, \nu, K, M)>0$ such that

$$
P\left(\|Y\|_{p-v a r ;[0, T]}>r\right) \leq \frac{1}{\eta} \exp \left(-\eta r^{2 / q}\right) \quad \text { for all } r \geq 0
$$

and the same holds for $\|Z\|_{p-v a r ;[0, T]}$ if $\nu \geq\|\varphi\|_{L i p^{\gamma-1}}$ instead. In particular, $\|Y\|_{p-v a r ;[0, T]}$ and $\|Z\|_{p-v a r ;[0, T]}$ have finite exponential moments as long as $q<2$. 
Proof. From 5 we know that there is a $\alpha=\alpha(\rho, p, M)$ such that

$$
P\left\{\|\mathbf{X}\|_{p-v a r ;[0, T]}^{p} \leq \frac{\alpha}{2^{p}[p]}\right\} \geq \frac{1}{2} .
$$

Hence, by Corollary 2, applied with $\hat{a}=\Phi^{-1}\left(\frac{1}{2}\right)=0$, and the remark thereafter,

$$
P\left\{N_{\alpha,[0, T]}(\mathbf{X})>r\right\} \leq \exp \left\{-\frac{1}{2}\left(\frac{\alpha^{1 / p} r^{1 / q}}{c_{1}}\right)^{2}\right\} \quad \text { for all } r \geq 0
$$

with $c_{1}=c_{1}(p, q, K, M)$. Corollary 3 shows that there is a constant $c_{2}=c_{2}(p, q, K, M, \gamma, \nu)$ such that also

$$
P\left\{N_{\alpha,[0, T]}(\mathbf{Y})>r\right\} \leq c_{2} \exp \left\{-\frac{r^{2 / q}}{c_{2}}\right\} \quad \text { for all } r \geq 0 .
$$

From Lemma 4 we see that

$$
\|Y\|_{p-v a r ;[0, T]} \leq\|\mathbf{Y}\|_{p-v a r ;[0, T]} \leq \alpha^{1 / p}\left(N_{\alpha,[0, T]}(\mathbf{Y})+1\right)
$$

which shows the claim for $\|Y\|_{p-v a r ;[0, T]}$. The same holds true for $\|Z\|_{p-v a r ;[0, T]}$ by using Corollary 4

Remark 6. In the Brownian motion case $(q=1)$, we recover the well-known fact that solutions $Y$ of the Stratonovich SDE

$$
d Y=V(Y) \circ d B ; \quad Y_{0} \in \mathbb{R}^{e}
$$

have Gaussian tails at any fixed point $Y_{t}$ provided $V$ is sufficiently smooth. We also recover that the Stratonovich integral

$$
\int_{0}^{t} \varphi(B) \circ d B
$$

has finite Gaussian tails for every $t \geq 0$, $\varphi$ sufficiently smooth.

Proposition 4. Let $X$ be as in Proposition [3. Let $Y:[0, T] \rightarrow \mathbb{R}^{e}$ be the pathwise solution of the stochastic linear $R D E$

$$
d Y=V(Y) d \mathbf{X} ; \quad Y_{0} \in \mathbb{R}^{e}
$$

where $V=\left(V_{i}\right)_{i=1, \ldots, d}$ is a collection of linear vector fields of the form $V_{i}(z)=A_{i} z+b_{i}, A_{i}$ are $e \times e$ matrices and $b_{i} \in \mathbb{R}^{e}$. Then $\log \left(\|Y\|_{p-v a r ;[0, T]}\right)$ has a Weibull tail with shape $2 / q$. More precisely, if $K \geq\|\iota\|_{o p}, M \geq V_{\rho-v a r}\left(R ;[0, T]^{2}\right)$ and $\nu \geq \max _{i}\left(\left|A_{i}\right|+\left|b_{i}\right|\right)$ there is a constant $\eta=\eta(p, q, \rho, \nu, K, M)>0$ such that

$$
P\left(\log \left(\|Y\|_{p-v a r ;[0, T]}\right)>r\right) \leq \frac{1}{\eta} \exp \left(-\eta r^{2 / q}\right) \quad \text { for all } r \geq 0
$$

In particular, $\|Y\|_{p-v a r ;[0, T]}$ has finite $L^{s}$-moments for any $s>0$ provided $q<2$.

Proof. Same as for Proposition 3 using Corollary 5 .

Remark 7. In the case $q=1$, which covers Brownian driving signals, we have log-normal tails. This is in agreement with trivial examples such as the standard Black-Scholes model in which the stock price $S_{t}$ is log-normally distributed.

Remark 8. The same conclusion holds for unbounded vector fields as seen in remark 5 . 
6.2. The Jacobian of the solution flow for SDEs driven by Gaussian signals. Let $x:[0, T] \rightarrow$ $\mathbb{R}^{d}$ be smooth and let $V=\left(V^{1}, \ldots, V^{d}\right): \mathbb{R}^{e} \rightarrow \mathbb{R}^{e}$ be a collection of vector fields. We can interpret $V$ as a function $V: \mathbb{R}^{e} \rightarrow L\left(\mathbb{R}^{d}, \mathbb{R}^{e}\right)$ with derivative $D V: \mathbb{R}^{e} \rightarrow L\left(\mathbb{R}^{e}, L\left(\mathbb{R}^{d}, \mathbb{R}^{e}\right)\right) \cong$ $L\left(\mathbb{R}^{d}\right.$, End $\left.\left(\mathbb{R}^{e}\right)\right)$. It is well-known that for sufficiently smooth $V$, the ODE

$$
d y=V(y) d x
$$

has a solution for every starting point $y_{0}$ and the solution flow $y_{0} \rightarrow U_{t \leftarrow 0}\left(y_{0}\right)=y_{t}$ is (Fréchet) differentiable. We denote its derivative by $J_{t \leftarrow 0}^{x}\left(y_{0}\right)=\left.D U_{t \leftarrow 0}(\cdot)\right|_{\cdot=y_{0}}$. Moreover, for fixed $y_{0}$, the Jacobian $J_{t}=J_{t \leftarrow 0}^{x}\left(y_{0}\right)$ is given as the solution of the linear ODE

$$
d J_{t}=d M_{t} \cdot J_{t} ; \quad J_{0}=I d
$$

where $M_{t} \in \operatorname{End}\left(\mathbb{R}^{e}\right)$ is given by the integral

$$
M_{t}=\int_{0}^{t} D V\left(y_{s}\right) d x_{s}
$$

If $\mathbf{x}$ is a $p$-rough path, one proceeds in a similar fashion. First, in order to make sense of (6.1) if $\mathbf{x}$ and $\mathbf{y}$ are rough paths, one has to define the joint rough path $(\mathbf{x}, \mathbf{y})=\mathbf{z} \in C^{p-v a r}\left([0, T], G^{[p]}\left(\mathbb{R}^{d} \oplus \mathbb{R}^{e}\right)\right)$ first. To do so, one defines $\mathbf{z}$ as the solution of the full RDE

$$
d \mathbf{z}=\tilde{V}(\mathbf{z}) d \mathbf{x} ; \quad \mathbf{z}_{0}=\exp \left(0, y_{0}\right) .
$$

where $\tilde{V}=(I d, V)$. Then, one defines $\mathbf{M} \in C^{p-v a r}\left([0, T], G^{[p]}\left(\mathbb{R}^{e \times e}\right)\right)$ as the rough integral

$$
\mathbf{M}_{t}=\int_{0}^{t} \phi(z) d \mathbf{z}
$$

where $\phi: \mathbb{R}^{d} \oplus \mathbb{R}^{e} \rightarrow L\left(\mathbb{R}^{d} \oplus \mathbb{R}^{e}\right.$, End $\left.\left(\mathbb{R}^{e}\right)\right)$ is given by $\phi(x, y)\left(x^{\prime}, y^{\prime}\right)=D V(y)\left(x^{\prime}\right)$ for all $x, x^{\prime} \in \mathbb{R}^{d}$ and $y, y^{\prime} \in \mathbb{R}^{e}$. Finally, one obtains $J_{t}^{\mathbf{x}}=J_{t \leftarrow 0}^{\mathbf{x}}\left(y_{0}\right)$ as the solution of the linear RDE

$$
d J_{t}^{\mathbf{x}}=d \mathbf{M}_{t} \cdot J_{t}^{\mathbf{x}} ; \quad J_{0}=I d .
$$

All this can be made rigorous; for instance, see [12, Theorem 11.3. Next, we give an alternative proof of the main result of [4, slightly sharpened in the sense that we consider the $p$-variation norm instead of the supremum norm.

Proposition 5. Let $X$ be a centred Gaussian process in $\mathbb{R}^{d}$ with independent components and covariance $R_{X}$ of finite $\rho$-variation, $\rho<2$. Consider the Gaussian $p$-rough paths $\mathbf{X}$ for $p>2 \rho$ and assume that there is a continuous embedding

$$
\iota: H \hookrightarrow C^{q-v a r}
$$

where $\frac{1}{p}+\frac{1}{q}>1$. Then $\log \left(\left\|J_{\cdot \leftarrow 0}^{\mathbf{X}}\left(y_{0}\right)\right\|_{p-v a r ;[0, T]}\right)$ has a Weibull tail with shape $2 / q$. In particular, if $q<2$, this implies that $\left\|J_{\leftarrow \leftarrow 0}^{\mathbf{X}}\left(y_{0}\right)\right\|_{p-v a r ;[0, T]}$ has finite $L^{r}$-moments for any $r>0$.

Proof. From Corollary 2 we know that $N_{1,[0, T]}(\mathbf{X})$ has a Weibull tail with shape 2/q. Combining the Corollaries 3, 4 and 5 shows that there is a constant $C$ such that

$$
\log \left(N_{1,[0, T]}\left(\mathbf{J}_{\leftarrow \leftarrow 0}^{\mathbf{X}}\left(y_{0}\right)\right)+1\right) \leq C\left(N_{1 ;[0, T]}(\mathbf{X})+1\right) .
$$

From Lemma 4 we know that

$$
\left\|J_{\leftarrow \leftarrow 0}^{\mathbf{X}}\left(y_{0}\right)\right\|_{p-v a r ;[0, T]} \leq\left\|\mathbf{J}_{\cdot \leftarrow 0}^{\mathbf{X}}\left(y_{0}\right)\right\|_{p-v a r ;[0, T]} \leq N_{1,[0, T]}\left(\mathbf{J}_{\cdot \leftarrow 0}^{\mathbf{X}}\left(y_{0}\right)\right)+1 .
$$


6.3. An example from rough SPDE theory. In situations where one performs a change of measure to an equivalent measure on a path space, one often has to make sense of the exponential moments of a stochastic integral, i.e. to show that

$$
E\left(\exp \left\{\int G(X) d X+\int F(X) d t\right\}\right)
$$

is finite for a given process $X$ and some suitable maps $G$ and $F$. The second integral is often trivially handled (say, when $F$ is bounded) and thus take $F=0$ in what follows. Various situations in the literarture (e.g. [13, 7]) require to bound (6.2) uniformly over a family of processes, say $\left(X^{\varepsilon}: \varepsilon>0\right)$. We will see in this section that our results are perfectly suited for doing this.

In the following, we study the situation of [13, section 4 . Here $\psi^{\varepsilon}=\psi^{\varepsilon}(t, x ; \omega)$ is the stationary (in time) solution to the damped stochastic heat equation with hyper-viscosity of parameter $\varepsilon>0$,

$$
d \psi^{\epsilon}=-\epsilon^{2} \partial_{x x x x} \psi^{\epsilon} d t+\left(\partial_{x x}-1\right) \psi^{\epsilon} d t+\sqrt{2} d W_{t}
$$

where $W$ is space-time white noise, a cylindrical Wiener process over $L^{2}(\mathbb{T})$ where $\mathbb{T}$ denotes the torus, say $[-\pi, \pi]$ with periodic boundary conditions. Following [13] we fix $t$, so that the "spatial" interval $[-\pi, \pi]$ plays the role of our previous "time-horizon" $[0, T]$. Note that $x \mapsto \psi^{\epsilon}(x, t)$ is a centred Gaussian process on $\mathbb{T}$, with independent components and covariance given by

$$
E\left(\psi^{\epsilon}(x, t) \otimes \psi^{\epsilon}(y, t)\right)=R^{\epsilon}(x, y) I=K^{\epsilon}(x-y) I
$$

where $K_{\epsilon}(x)$ is proportional to

$$
\sum_{k \in \mathbb{Z}} \frac{\cos (k x)}{1+k^{2}+\epsilon^{2} k^{4}}
$$

As was pointed out by Hairer, it can be very fruitful in a non-linear SPDE context to consider $\lim _{\varepsilon \rightarrow 0} \psi^{\varepsilon}(t, \cdot, \omega)$ as random spatial rough path. To this end, it is stated (without proof) in 13 that the covariance of $\psi^{\varepsilon}$ has fnite $\rho$-variation in $2 \mathrm{D}$ sense, $\rho>1$, uniformly in $\varepsilon$. In fact, we can show something slightly stronger. Following [13, $\psi^{\varepsilon}$ is $C^{1}$ in $x$ for every $\varepsilon>0$, and can be seen as $p$-rough path, any $p>2$, when $\varepsilon=0$.

Lemma 8. The map $\mathbb{T}^{2} \ni(x, y) \mapsto R^{\epsilon}(x, y)$ has finite 1-variation in $2 D$ sense, uniformly in $\varepsilon$. That is,

$$
M:=\sup _{\varepsilon \geq 0} V_{1-\operatorname{var}}\left(R^{\varepsilon} ; \mathbb{T}^{2}\right)<\infty .
$$

Proof. By lower semi-continuity of variation norms under pointwise convergence, it suffices to consider $\varepsilon>0$. (Alternatively, the case $\varepsilon=0$ is treated explicitly in [13]). We then note that

$$
\sum_{k \in \mathbb{Z}} \frac{\cos (k x)}{1+\epsilon^{2} k^{2}}=\frac{\pi \cosh \left(\frac{1}{\epsilon}(|x|-\pi)\right)}{\epsilon \sinh \left(\frac{\pi}{\epsilon}\right)}
$$

in $L^{2}(\mathbb{T})$ as may be seen by Fourier expansion on $[-\pi, \pi]$ of the function $x \mapsto \cosh \left(\frac{1}{\epsilon}(|x|-\pi)\right)$. Since $\left|\partial_{x, y} R_{\epsilon}(x, y)\right|=\left|K_{\epsilon}^{\prime \prime}(x-y)\right|$ we have

$$
V_{1-v a r}\left(R^{\varepsilon} ; \mathbb{T}^{2}\right)=\int_{\mathbb{T}^{2}}\left|\partial_{x, y} R_{\epsilon}(x, y)\right| d x d y=\int_{\mathbb{T}^{2}}\left|K_{\epsilon}^{\prime \prime}(x-y)\right| d x d y
$$


On the other hand,

$$
\begin{aligned}
\left|K_{\epsilon}^{\prime \prime}(x)\right| & \leq\left|\sum_{k \in \mathbb{Z}}\left(\frac{1}{1+\epsilon^{2} k^{2}}-\frac{k^{2}}{1+k^{2}+\epsilon^{2} k^{4}}\right) \cos (k x)\right|+\frac{\pi \cosh \left(\frac{1}{\epsilon}(|x|-\pi)\right)}{\epsilon \sinh \left(\frac{\pi}{\epsilon}\right)} \\
& =\left|1+\sum_{k \neq 0} \frac{1}{k^{2}} \frac{\cos (k x)}{\left(1+\epsilon^{2} k^{2}\right)\left(1 / k^{2}+1+\epsilon^{2} k^{2}\right)}\right|+\frac{\pi \cosh \left(\frac{1}{\epsilon}(|x|-\pi)\right)}{\epsilon \sinh \left(\frac{\pi}{\epsilon}\right)} \\
& \leq 1+\sum_{k \neq 0} \frac{1}{k^{2}}+\frac{\pi \cosh \left(\frac{1}{\epsilon}(|x|-\pi)\right)}{\epsilon \sinh \left(\frac{\pi}{\epsilon}\right)} \\
& \leq 1+\frac{\pi^{2}}{3}+\frac{\pi \cosh \left(\frac{1}{\epsilon}(|x|-\pi)\right)}{\epsilon \sinh \left(\frac{\pi}{\epsilon}\right)}
\end{aligned}
$$

Hence

$$
\int_{\mathbb{T}^{2}}\left|K_{\epsilon}^{\prime \prime}(x-y)\right| d x d y \leq(2 \pi)^{2}\left(1+\frac{\pi^{2}}{3}\right)+\frac{\pi}{\epsilon \sinh \left(\frac{\pi}{\epsilon}\right)} \int_{\mathbb{T}^{2}} \cosh \left(\frac{1}{\epsilon}(|x-y|-\pi)\right) d x d y .
$$

We leave to the reader to see that the final integral is bounded, independent of $\varepsilon$. For instance, introduce $z=x-y$ as new variable so that only

$$
\begin{aligned}
& \frac{\pi}{\epsilon \sinh \left(\frac{\pi}{\epsilon}\right)} \int_{-2 \pi}^{2 \pi} \cosh \left(\frac{1}{\epsilon}(|z|-\pi)\right) d z \\
= & 4 \frac{\pi}{\epsilon \sinh \frac{\pi}{\epsilon}} \int_{0}^{\pi} \cosh \left(\frac{z}{\epsilon}\right) d z
\end{aligned}
$$

needs to be controlled. Using $\cosh \approx \sinh \approx \exp$ for large arguments (or integrating explicitly ...) we get

$$
\begin{aligned}
\frac{1}{\epsilon \sinh \left(\frac{\pi}{\epsilon}\right)} \int_{0}^{\pi} \cosh \left(\frac{z}{\epsilon}\right) d z & \approx \int_{0}^{\pi} \frac{\exp \left(\frac{z}{\epsilon}\right)}{\epsilon \exp \left(\frac{\pi}{\epsilon}\right)} d z \\
& =\frac{\exp \left(\frac{\pi}{\varepsilon}\right)-1}{\exp \left(\frac{\pi}{\epsilon}\right)} \\
& \leq 1
\end{aligned}
$$

We then have the following sharpening of [13], Theorem 5.1.

Theorem 2. Fix $\gamma>2$ and $p \in(2, \gamma)$. Assume $G=\left(G_{i}\right)_{i=1, \ldots, d}$ is a collection of Lip ${ }^{\gamma-1}\left(\mathbb{R}^{d}, \mathbb{R}^{e}\right)$ maps. Then for some constant $\eta=\eta\left(\gamma, p,\|G\|_{L i p^{\gamma-1}}\right)>0$ we have the uniform estimate

$$
\sup _{t \in[0, \infty)} \sup _{\varepsilon \geq 0} E\left\{\exp \left(\eta\left|\int_{\mathbb{T}} G\left(\psi^{\epsilon}(x, t)\right) d_{x} \psi^{\epsilon}(x, t)\right|^{2}\right)\right\}<\infty .
$$

(When $\varepsilon>0, \psi^{\epsilon}$ is known to be $C^{1}$ in $x$ so that we deal with Riemann-Stieltjes integrals, when $\varepsilon=0$, the integral is understood in rough path sense.) 
Proof. By stationarity in time of $\psi^{\epsilon}(\cdot, t)$, uniformity in $t$ is trivial. Note that the Riemann-Stieltjes integral

$$
\int_{\mathbb{T}} G\left(\psi^{\epsilon}(x, t)\right) d_{x} \psi^{\epsilon}(x, t)
$$

can also be seen as rough integral where the integrator is given by the "smooth" rough path $\left(\psi^{\varepsilon}, \int \psi^{\varepsilon} \otimes d_{x} \psi^{\varepsilon}\right)$ when $\varepsilon>0$. For $\varepsilon=0$, the above integral is a genuine rough integral, the existence of a canonical lift of $\psi^{0}(\cdot, t)$ to a geometric rough path is a standard consequence (cf. [11, 12]) of finite 1-variation of $R^{0}$, the covariance function of $\psi^{0}(\cdot, t)$. After these remarks,

$$
\sup _{\varepsilon \geq 0} E\left\{\exp \left(C\left|\int_{\mathbb{T}} G\left(\psi^{\epsilon}(x, t)\right) d_{x} \psi^{\epsilon}(x, t)\right|^{2}\right)\right\}<\infty
$$

is an immediate application of Lemma 8 and Proposition 3

Acknowledgement 1. It is a pleasure to thank M. Hairer, H. Weber and T. Lyons for related discussions. P.K. Friz has received funding from the European Research Council under the European Union's Seventh Framework Programme (FP7/2007-2013) / ERC grant agreement nr. 258237. S. Riedel is supported by an IRTG (Berlin-Zurich) PhD-scholarship.

\section{REFERENCES}

[1] Baudoin, F.; Hairer, M.: A version of Hörmander's theorem for the fractional Brownina motion, Probab. Theory Related Fields 139, 373-395, 2007

[2] Cass, T.; Friz, P.: Densities for rough differential equations under Hörmander's condition, Ann. of Math. (2), 171(3):2115-2141, 2010

[3] Cass, T.; Friz, P.; Victoir, N.: Non-degeneracy of Wiener functionals arising from rough differential equations, Trans. Amer. Math. Soc., 361(6):3359-3371, 2009

[4] Cass, T.; Litterer, C.; Lyons, T.: Integrability estimates for Gaussian rough differential equations, arXiv:1104.1813v4, 2011

[5] Cass, T.; Lyons, T.: Evolving communities and individual preferences, Preprint, 2010

[6] Coutin, L.; Qian, Z.: Stochastic analysis, rough path analysis and fractional Brownian motion, Probab. Theory Related Fields 122, 108-140, 2002

[7] Crisan, D.; Diehl, J.; Friz, P.; Oberhauser, H.: Robust filterin: correlated noise and multidimensional observation, arXiv:1201.1858v1, 2012

[8] Driscoll, P.: Smoothness of Density for the Area Process of Fractional Brownian Motion, arXiv:1010.3047v1, 2010

[9] Friz, P.; Oberhauser, H.: Rough paths limits of the Wong-Zakai type with a modified drift term, J. Funct. Anal., 256(10):3236-3256, 2009

[10] Friz, P.; Oberhauser, H.: A generalized Fernique theorem and applications, Proceedings of the American Mathematical Society, 138, 3679-3688, 2010

[11] Friz, P.; Victoir, N.: Differential equations driven by Gaussian signals, Annales de l'Institut Henri Poincaré (B) Probability and Statistics, Vol. 46, No. 2, 369-413, 2010

[12] Friz, P.; Victoir, N.: Multidimensional Stochastic Processes as Rough Paths, Cambridge Studies in Advanced Mathematics Vol 120, Cambridge University Press, 2010

[13] Hairer, M.: Rough stochastic PDEs, Comm. PureAppl. Math. 64, no.11, 1547-1585, 2011

[14] Hairer, M.; Pillai, N. S.: Regularity of Laws and Ergodicity of Hypoelliptic SDEs Driven by Rough Paths, arXiv:1104.5218v1, 2011

[15] Inahama, Y.: A moment estimate of the derivative process in rough path theory, arXiv:1007.4651v1, 2010

[16] Ledoux, M.: Isoperimetry and Gaussian Analysis, Lectures on Probability Theory and Statistics (Saint-Flour, 1994), Vol. 1648 of Lecture Notes in Mathematics, p. 165-294, Springer, 1996

[17] Ledoux, M.; Qian, Z.; Zhang, T.: Large deviations and support theorem for diffusion processes via rough paths, Stoch. Proc. Appl. 102, no. 2, 265-283, 2002

[18] Lejay, A.: On rough differential equations, Electronic Journal of Probability [Online], Vol. 14, 2009

[19] Lyons, T.: Differential equations driven by rough signals, Rev. Mat. Iberoamericana 14, no. 2, 215-310, 1998 
[20] Lyons, T.; Qian, Z.: System Control and Rough Paths, Oxford University Press, 2002

[21] Lyons, T.; Caruana, M.; Lévy, T.: Lectures on Probability Theory and Statistics (Saint-Flour, 1996), Vol. 1908 of Lecture Notes in Mathematics, Springer, 2007

[22] Lyons, T.; Zeitouni, O.: Conditional exponential moments for iterated Wiener integrals, Ann. Probab. 27, no. 4, 1738-1749, 1999

[23] Malliavin, P.: Stochastic Analysis, Volume 313 of Grundlehren der Mathematischen Wissenschaften [Fundamental Principles of Mathematical Sciences], Springer, 1997

[24] Millet, A.; Sanz-Solé, M.: Large deviations for rough paths of the fractional Brownian motion, Annales de l'Institut Henri Poincaré (B) Probability and Statistics, Vol. 42, 245-271, 2006

TU AND WIAS BERLin (First AND CORRESPONDING AUthor, FRIZ@MATH.TU-BERLin.de), TU BerLin (SECOND AUTHOR) 